\title{
Aleitamento Natural, Aleitamento Artificial, Hábitos de Sucção e Maloclusões em Pré-escolares Brasileiros
}

\author{
Breast-feeding, bottle-feeding, sucking habits and malocclusion in \\ brazilian preschool children
}

\author{
Alessandro Leite-Cavalcanti, Priscila K. Medeiros-Bezerra y Cristiano Moura
}

Universidade Estadual da Paraíba, Campina Grande, Paraíba, Brasil. dralessandro@ibest.com.br; priscillakelly_pk@hotmail.com; moura01@uol.com.br.

Recibido 3 Noviembre 2006/Enviado para Modificación 31 Marzo 2007/Aceptado 30 Abril 2007

\section{RESUMO}

Objetivo O objetivo deste estudo foi verificar a prevalência de hábitos de sucção nutritivos (aleitamento natural e artificial) e não nutritivos e a presença de maloclusão em préescolares brasileiros.

Material e Métodos Este estudo transversal examinou 342 crianças (196 meninos e 146 meninas) entre 3 e 5 anos de idade em Campina Grande, Brasil. Os dados foram coletados através de entrevistas com as mães das crianças e o exame clínico realizado por um examinador calibrado (kappa $=0,86$ ). A análise estatística foi executada através do uso do programa Epi Info 3.3 e do Teste do Qui-quadrado a um nível de significância de 0,05 .

Resultados A prevalência de hábitos de sucção foi elevada em todas as faixas etárias, variando de $70 \%$ a 77,4 \%. Maloclusões estavam presentes em $87 \%$, sucção de chupeta em $84,8 \%$ e sucção digital em 7,2 \%. Aproximadamente $84,2 \%$ das crianças tinham história de alimentação artificial e 79,9 \% delas apresentavam maloclusão quando do exame. Existiram diferenças significativas entres as variáveis: hábitos de sucção e presença de maloclusão; tempo de alimentação natural e presença de hábitos de sucção; tempo de alimentação natural e presença de maloclusão; tipo de alimentação e hábitos de sucção; e tipo de alimentação e presença de maloclusão.

Conclusão $A$ incidência de sucção de chupeta foi maior do que a de sucção digital entre os pré-escolares brasileiros. A freqüência de hábitos de sucção foi mais elevada entre as crianças com alimentação artificial do que nas crianças com alimentação natural. A relação entre a presença de hábitos de sucção e de maloclusão foi estatisticamente significante.

Palavras Chave: Epidemiologia, amamentação, maloclusão, criança (fuente: DeCS, BIREME).

\section{ABSTRACT}

Objective This study was aimed at verifying the prevalence of nutritive (breast-feeding 
and bottle-feeding) and non-nutritive sucking habits and the presence of malocclusion in Brazilian preschool children.

Material and Methods The study was a cross-sectional oral health survey of 342 children (196 boys and 146 girls) between the ages of 3 and 5 in Campina Grande, Brazil. The data was collected by interviews with the children's mothers or minders and by clinical examinations carried out by a calibrated examiner (kappa $=0,86)$. Descriptive statistics using the EPI-INFO Program (version 3.3) and Chi-square test at 0.05 probability level were produced.

Results Sucking habit prevalence was very high in all groups, ranging from $70 \%$ to 77,4 $\%$. Malocclusion was present in $87 \%$, dummy use in $84,8 \%$ and finger-sucking in $7,2 \%$. About $84,2 \%$ of the children had a history of bottle-feeding and $79,9 \%$ showed some evidence of malocclusion at the time of dental examination. There were significant differences for the following variables: sucking habits and malocclusion; breast-feeding time and sucking habits; breast-feeding time and malocclusion; type of feeding and sucking habits; and type of feeding and malocclusion.

Conclusion Dummy-sucking incidence was higher than that of finger-sucking in Brazilian children. Sucking habit incidence was higher in bottle-fed children than in breast-fed children. The relationship between incidence of habits and malocclusion was statistically significant.

Key Words: Epidemiology, breast-feeding, malocclusion, child (source: MeSH, NLM).

$\mathrm{H}$ ábito é o costume ou a prática adquirida pela repetição freqüente de um mesmo ato, que a princípio se faz de forma consciente e, posteriormente, de modo inconsciente. Arespiração nasal, a mastigação e a deglutição são considerados hábitos fisiológicos e funcionáis.Entretanto, a suç̧ão digital, de chupeta, mamadeira e a respiração bucal, dentre outros, são considerados hábitos não fisiológicos, portanto, deletérios ou parafuncionais (1).

Os principais hábitos que suscitam deformidades na oclusão são: onicofagia, bruxismo, respiração bucal, interposição lingual, morder objetos, morder lábios, além dos mais típicos hábitos deletérios de suç̧ão de dedo, chupeta e mamadeira $(2,3)$. Estes últimos são de fácil aquisição e tendem a perdurar, principalmente em crianças que não receberam, ou mesmo obtiveram de forma insatisfatória uma amamentação natural nos seis primeiros meses de vida $(3,4)$.

A amamentação natural (no seio) e artificial (na mamadeira) envolve vários músculos orofaciais, conduzindo a distintos efeitos no crescimento harmônico dos maxilares e dos arcos dentais (5). A sucção é um reflexo inato que proporciona ao recém nascido a sobrevivência e estabelece vínculo afetivo com a mãe durante a amamentação. 
A amamentação natural durante os seis primeiros meses de vida é importante não apenas para a nutrição, mas também para o fortalecimento do sistema imunológico e para o correto desenvolvimento da oclusão decídua (6). As funções realizadas através da amamentação promovem estímulos neurais adequados ao crescimento ósseo e muscular para prevenir maloclusões por hipodesenvolvimento (7).

O desmame precoce pode levar à ruptura do desenvolvimento motor-oral adequado, provocando alterações na postura e força dos órgãos fonoarticulatórios e prejudicando as funções de mastigação, deglutição, respiração e articulação dos sons da fala. A falta da sucção fisiológica ao peito pode interferir no desenvolvimento motor-oral, possibilitando a instalação de má oclusão, respiração oral e alteração motora-oral (6).

Quando as crianças são alimentadas por mamadeiras, é minimizado o trabalho da musculatura perioral. Com menor número de sucções e o êxtase emocional não atingido, a criança passa a buscar substitutos como o dedo e/ou a chupeta $(4,7)$. No que concerne à utilização da chupeta, a sua oferta à criança pelos pais ou responsáveis tem por propósito trazer conforto e tranqüilidade (8). É sabido, porém, que gravidade dos problemas advindos do hábito de sucção depende também da duração, freqüência e intensidade de seu uso, como também da predisposição individual, com presença ou não de doenças somáticas $(7,9)$.

Diversos estudos $(1,2,4,5,10-18)$ estabeleceram a relação entre a presença de hábitos parafuncionais e aleitamento com alterações oclusais em crianças. Vários autores sugeriram que a sucção não nutritiva (chupeta ou dedo) pode ser responsável pelo surgimento de maloclusões na infância, principalmente a mordida aberta anterior (1,19-23).

Portanto, este estudo objetivou analisar a prevalência de hábitos de sucção e sua associação com o tipo de aleitamento e a presença de maloclusão em pré-escolares brasileiros.

\section{METODOLOGIA}

Área do Estudo

A pesquisa foi desenvolvida no município de Campina Grande, estado da Paraíba, localizada no Agreste da Borborema, nordeste do Brasil. A população estimada compreende 376132 habitantes (24). 
Delineamento

Realizou-se um estudo epidemiológico, do tipo transversal, descritivo-analítico, em pré-escolares regularmente matriculados nas creches públicas municipais de Campina Grande, Brasil. Previamente à realização do trabalho, o estudo foi registrado no SISNEP (Sistema Nacional de Informação sobre Ética em Pesquisa envolvendo Seres Humanos, protocolo CAAE 0988) e aprovado pelo Comitê de Ética em Pesquisa da Universidade Estadual da Paraíba. A coleta de dados foi realizada no período de outubro de 2005 a abril de 2006.

O universo abrangeu 2464 pré-escolares entre 3 e 5 anos de idade, regularmente matriculadas nas 21 creches públicas municipais, conforme dados fornecidos pela Secretaria da Educação. A amostra foi do tipo probabilístico, sendo composta por 342 crianças (196 meninos e 146 meninas), os quais foram divididos em 3 grupos, de acordo com a faixa etária: G1 (3 anos) com 127 (37,1\%); G2 (4 anos) com 115 (33,6\%) e G3 (5 anos) com 100 (29,2\%) préescolares.

O instrumento de pesquisa consistiu de um questionário específico abordando as seguintes variáveis: gênero e faixa etária, tipo de aleitamento, presença e tipo de hábito deletério, freqüência do hábito e presença de maloclusão.

Os pais e responsáveis foram informados sobre os benefícios do estudo e após a assinatura do Termo de Consentimento Livre e Esclarecido realizou-se a entrevista e o exame da oclusão. Um único examinador calibrado (kappa = 0,86), portando os aparatos necessários ao correto controle de infecção, procedeu ao exame clínico, o qual foi feito no próprio ambiente da creche, sob iluminação natural, através da inspeção visual.

Os dados obtidos foram organizados por intermédio do software Epi-INFO 2003 e submetidos à análise estatística através do Teste do Qui-Quadrado. A codificação e digitação das respostas ficaram sob a responsabilidade do pesquisador principal a fim de se evitar inconsistência ou perda de informações.

\section{RESULTADOS}

O percentual de crianças que possuíam hábitos de suç̧ão correspondeu a 73,4 \%, ou seja, 251 crianças, não sendo observadas diferenças estatisticamente significantes entre os gêneros ( $\mathrm{p}>0,05)$, conforme descrito na Tabela 1. 
Quando se avaliou a presença de hábitos de acordo com a idade, verificouse que o maior percentual foi apresentado pelas crianças de 4 anos $(77,4 \% n=88)$, seguido dos pré-escolares com 3 anos $(72,4 \%$ - $n=92)$ e das crianças com 5 $\operatorname{anos}(70 \% \mathrm{n}=70)$.

Tabela 1. Distribuição dos pré-escolares segundo o gênero e a presença

\begin{tabular}{ccccccc}
\multicolumn{7}{c}{ Presença de Hábitos } \\
Gênero & \multicolumn{3}{c}{ SIM NÃO } & \multicolumn{2}{c}{ Total } \\
& $n$ & $\%$ & $n$ & $\%$ & $n$ & $\%$ \\
\hline Masculino & 137 & 69,9 & 59 & 30,1 & 196 & 100 \\
Feminino & 114 & 78,1 & 32 & 21,9 & 146 & 100 \\
Total & 251 & 73,4 & 91 & 26,6 & 342 & 100 \\
\hline
\end{tabular}

Ao se analisar o número de hábitos bucais presentes por criança, verificouse que 79,3 \% (n=199) apresentavam um único tipo, 17,1 \% (n=43) possuíam dois diferentes tipos de hábitos e 3,6\% (n=9) portavam 3 tipos de hábitos bucais diferentes.

Examinando-se os tipos de hábitos existentes, constatou-se que a sucção de chupeta mostrou-se como o mais freqüente, ocorrendo em $84,8 \%$ das crianças $(n=213)$, apresentando-se isolado ou associado com um ou mais diferentes hábitos. Asuç̧ão digital foi diagnosticada em 7,2 \% (n=18), enquanto a onicofagia em 4,4\% (n=11) das crianças. Os demais hábitos (morder objetos e interposição lingual) foram relatados associados apresentando-se em freqüências menores e totalizaram 3,6 \%.

Ao se associar a presença dehábitos bucais com a existência de maloclusões, verificou-se que das 251 crianças portadoras de hábitos, 220 (87\%) apresentavam maloclusão, sendo este resultado estatisticamente significante $(\mathrm{p}<0,001)$ (Tabela 2).

Tabela 2. Associação entre presença de hábito de sucção e existência

\begin{tabular}{|c|c|c|c|c|c|}
\hline \multirow{4}{*}{$\begin{array}{l}\text { Hábitos de } \\
\text { Sucção }\end{array}$} & \multirow{2}{*}{\multicolumn{4}{|c|}{ Presença de Maloclusão }} & \multirow{4}{*}{ OR (IC95\%) } \\
\hline & & & & & \\
\hline & \multicolumn{2}{|c|}{ SIM } & \multicolumn{2}{|c|}{ NÃO } & \\
\hline & $\mathrm{N}$ & $\%$ & $\mathrm{n}$ & $\%$ & \\
\hline Presente & 220 & 87,0 & 31 & 34,9 & 1,0 \\
\hline Ausente & 33 & 13,0 & 58 & 65,1 & 12,5 \\
\hline Total & 253 & 100,0 & 89 & 100,0 & $(7,05-22,03)$ \\
\hline
\end{tabular}

O aleitamento natural foi verificado em 84,8 \% $(n=290)$ das crianças, das quais 40,9\% (n=140) foram amamentadas até os seis meses de vida. A análise 
bivariada entre o aleitamento natural e a existência de hábitos bucais, conforme demonstrado na Tabela 3 revelou que das 70 crianças que foram aleitadas ao seio por um período igual ou maior que 19 meses, 65,7 \% $(n=46)$ não apresentavam hábitos bucais deletérios $(\mathrm{p}<0,01)$.

Tabela 3. Distribuição dos pré-escolares segundo o tempo de aleitamento natural e a presença de hábitos de sucção

\begin{tabular}{|c|c|c|c|c|c|c|c|}
\hline \multirow{3}{*}{$\begin{array}{c}\text { Tempo de } \\
\text { Aleitamento natural } \\
\text { (meses) }\end{array}$} & \multicolumn{4}{|c|}{ Presença de hábitos } & \multirow{2}{*}{\multicolumn{2}{|c|}{ Total }} & \multirow[b]{3}{*}{$p^{*}$} \\
\hline & \multicolumn{2}{|c|}{$\operatorname{sim}$} & \multicolumn{2}{|c|}{ Não } & & & \\
\hline & $\mathrm{n}$ & $\%$ & $\mathrm{n}$ & $\%$ & $\mathrm{~N}$ & $\%$ & \\
\hline Até 6 meses & 144 & 84,7 & 26 & 15,3 & 170 & 100,0 & \multirow{4}{*}{$p<0,01$} \\
\hline De 7 a 18 meses & 37 & 74,0 & 13 & 26,0 & 50 & 100,0 & \\
\hline 19 meses ou mais & 24 & 34,3 & 46 & 65,7 & 70 & 100,0 & \\
\hline Total & 205 & 70,7 & 85 & 29,3 & 290 & 100,0 & \\
\hline
\end{tabular}

Ao estabelecer uma associação entre a presença de maloclusão com o tempo de aleitamento (Tabela 4), pode-se observar que as crianças que foram amamentadas por um período igual ou inferior a 6 meses apresentaram maior freqüência de maloclusão (82,4 \%), quando comparadas aquelas que foram amamentadas por um período igual ou superior a 19 meses (45,7 \%), sendo estes resultados estatisticamente significantes.

Tabela 4. Associação entre a presença de maloclusão e a variação de tempo de

\begin{tabular}{|c|c|c|c|c|c|c|c|}
\hline \multirow{3}{*}{$\begin{array}{c}\text { Tempo de } \\
\text { Aleitamento natural } \\
\text { (meses) }\end{array}$} & \multicolumn{4}{|c|}{ Presença de maloclusão } & \multirow{2}{*}{\multicolumn{2}{|c|}{ Total }} & \multirow[b]{3}{*}{$\mathrm{p}^{*}$} \\
\hline & \multicolumn{2}{|c|}{ Sim } & \multicolumn{2}{|c|}{ Não } & & & \\
\hline & $\mathrm{n}$ & $\%$ & $\mathrm{n}$ & $\%$ & $n$ & $\%$ & \\
\hline Até 6 meses & 140 & 82,4 & 30 & 17,6 & 170 & 100 & \\
\hline De 7 a 18 meses & 36 & 72,0 & 14 & 28,0 & 50 & 100 & \\
\hline 19 meses ou mais & 32 & 45,7 & 38 & 54,3 & 70 & 100 & \\
\hline Total & 208 & 71,7 & 82 & 28,3 & 290 & 100 & $p<0,01$ \\
\hline
\end{tabular}

A análise bivariada entre o tipo de aleitamento e a existência de hábitos bucais deletérios (Tabela 5) mostrou que 205 (70,7 \%) das 290 crianças que foram amamentadas (aleitamento natural) apresentaram hábitos $(\mathrm{p}<0,05)$, enquanto aquelas crianças não receberam aleitamento natural, 88,5 \% (n=46) possuíam hábitos bucais $(\mathrm{p}<0,05)$.

Ainda com relação ao tipo de aleitamento (Tabela 5), 233 crianças (80,9 $\%$ receberam aleitamento artificial (mamadeira) e apresentaram um ou mais tipos de hábitos bucais. Contudo, entre aquelas que não receberam aleitamento 
artificial, apenas 18 (33,3 \%) desenvolveram hábitos, sendo esses resultados estatisticamente significantes $(\mathrm{p}<0,05)$.

Tabela 5. Distribuição dos pré-escolares segundo o tipo de aleitamento e a presença de hábitos de sucção

\begin{tabular}{ccccccccc}
\hline \multicolumn{1}{c}{ Tipo de Aleitamento } & \multicolumn{3}{c}{ Presença de hábitos } & \multicolumn{2}{c}{ Total } & \multirow{2}{*}{ * $^{*}$} \\
& & $\mathrm{n}$ & $\%$ & $\mathrm{n}$ & $\%$ & $\mathrm{n}$ & $\%$ & \\
\hline Natural & Sim & 205 & 70,7 & 85 & 29,3 & 290 & 100 & \multirow{2}{*}{0} \\
& Não & 46 & 88,5 & 6 & 11,5 & 52 & 100 & $\mathrm{p}<0,05$ \\
Artificial & Sim & 233 & 80,9 & 55 & 9,1 & 288 & 100 & \\
& Não & 18 & 33,3 & 36 & 66,7 & 54 & 100 & $\mathrm{p}<0,05$ \\
\hline * Qui-quadrado & & & & & & & &
\end{tabular}

Quanto à forma de aleitamento e sua relação com a presença de maloclusão, a Tabela 6 ilustra a situação na qual, das 290 crianças que foram aleitadas no seio $208(71,7 \%)$ desenvolveram maloclusão $(p<0,05)$. No que concerne ao aleitamento artificial (mamadeira), 79,9\% $(\mathrm{n}=230)$ das crianças que receberam este tipo de aleitamento, desenvolveram algum tipo de maloclusão, sendo este resultado estatisticamente significante $(\mathrm{p}<0,001)$.

Tabela 6. Distribuição dos pré-escolares segundo o tipo de aleitamento e a presença de maloclusão

\begin{tabular}{|c|c|c|c|c|c|c|c|c|c|}
\hline \multicolumn{2}{|c|}{ Tipo de Aleitamento } & \multicolumn{8}{|c|}{ Presença de maloclusão } \\
\hline & & \multicolumn{2}{|c|}{ Sim } & \multicolumn{2}{|c|}{ Não } & \multicolumn{2}{|c|}{ Total } & \multirow[b]{2}{*}{$p^{*}$} & \multirow[b]{2}{*}{ OR (IC95\%) } \\
\hline & & $n$ & $\%$ & $n$ & $\%$ & $n$ & $\%$ & & \\
\hline \multirow[b]{2}{*}{ Natural } & Sim & 208 & 71,7 & 82 & 28,3 & 290 & 100 & & \multirow{4}{*}{$\begin{array}{c}1 \\
5,34 \\
(2,89-9,85)\end{array}$} \\
\hline & Não & 45 & 86,5 & 7 & 13,5 & 52 & 100 & $p<0,05$ & \\
\hline \multirow[b]{2}{*}{ Artificial } & Sim & 230 & 79,9 & 58 & 20,1 & 288 & 100 & \multirow[b]{2}{*}{$p<0,001$} & \\
\hline & Não & 23 & 42,6 & 31 & 57,4 & 54 & 100 & & \\
\hline
\end{tabular}

\section{DISCUSSÃO}

Os hábitos bucais parafuncionais podem alterar o desenvolvimento normal do sistema estomatognático, devido a um desequilíbrio entre as forças musculares externas e o internas, produzindo uma deformação óssea, como a pressão do dedo na sucção ou a interposição de outros objetos como a chupeta $(1,25)$.

Este estudo revelou que 73,4 \% das crianças examinadas possuíam um ou mais tipos de hábitos bucais (Tabela 1), valor este muito superior aos 29,7 \% que foram encontrados na Índia (12), aos 34,8 \% no Brasil (14), aos 35,6 \% em Hong Kong (26) e aos $66 \%$ encontrados em crianças chilenas (1). Semelhante ao descrito na literatura $(20,23)$, não foram observadas diferenças estatísticas entre os gêneros. 
Quanto ao número de hábitos bucais, 20,7 \% dos pré-escolares apresentavam dois ou mais tipos de hábitos. Vários autores $(9,24,27)$ afirmam ser a sucção de chupeta o hábito mais comum entre as crianças, fato este confirmado nesta pesquisa, onde $84,9 \%$ das crianças possuíam este hábito. Foi encontrada uma associação positiva entre a presença de hábito bucal e a existência de maloclusão, sendo demonstrado que das crianças portadoras de hábitos, 87 \% apresentavam maloclusão, resultado este semelhante ao reportado por estudo prévio (23). É importante salientar que as crianças com hábitos bucais apresentam 12 vezes mais chance $(O . R .=12,47)$ de desenvolver maloclusão do que as crianças que não têm hábitos (Tabela 2).

A maioria das crianças recebeu aleitamento natural (84,8 \%), valor este superior aos $73 \%$ relatados na Austrália (28), porém inferior aos $98 \%$ encontrados na Turquia (4). A presente pesquisa revelou também que 70,7 \% das crianças que haviam recebido aleitamento natural possuíam hábitos bucais parafuncionais. Quando se analisa o período de tempo de aleitamento e a presença de hábito, encontrou-se que quanto menor o tempo de aleitamento natural maior a prevalência de hábitos bucais (Tabela 3), concordando com a literatura (16). Dessa forma, crianças com menor tempo de aleitamento materno desenvolvem, com maior freqüência, hábitos bucais deletérios, possuindo um risco relativo sete vezes superiores com relação àquelas aleitadas no seio por um período de, no mínimo, seis meses (2).

Fato semelhante foi observado quando da associação entre o tempo de aleitamento e presença de maloclusão. Nas crianças que foram amamentadas por um período de tempo igual ou inferior a 6 meses, 82,4 \% possuíam maloclusão, enquanto aquelas que foram amamentadas por um período de 19 meses ou mais, 45,7 \% tinham maloclusão. Na Itália, um estudo revelou um percentual de $32 \%$ de alterações na oclusão entre as crianças que haviam recebido aleitamento natural (5). Outro estudo demonstrou que crianças com mordida cruzada posterior apresentavam períodos de amamentação mais curtos quando comparadas àquelas crianças que não desenvolveram essa condição (13).

Ao se examinar a associação entre o tipo de aleitamento (se natural ou artificial) e presença de hábitos, percebe-se que a frequêencia de hábitos foi maior naquelas das crianças que receberam aleitamento artificial (80,9 \%) quando comparadas com aquelas que receberam aleitamento natural (70,7 \%) (Tabela 5), concordando, portanto, com a literatura que diz ser a incidência de 
hábitos bucais mais elevada em crianças que se alimentam com mamadeira (17). As crianças aleitadas com mamadeira por mais de um ano apresentam quase dez vezes mais risco de terem hábitos bucais viciosos do que aquelas que nunca utilizaram essa forma de aleitamento (2).

Quanto à forma de aleitamento e sua relação com a presença de maloclusão, crianças que receberam aleitamento artificial apresentaram uma maior prevalência (Tabela 6), existindo 5 vezes mais chances de desenvolver maloclusão entre os que foram aleitados artificialmente. Um estudo anterior encontrou uma prevalência de $41,0 \%$ de alterações oclusais em crianças com aleitamento artificial (5).

A introdução precoce do aleitamento artificial indica um padrão de baixoimpacto na atividade muscular, podendo interferir com o desenvolvimento normal dos rebordos alveolares e do palato duro, e conseqüentemente conduzir a uma maloclusão (13).

O conhecimento sobre a etiologia da maloclusão é essencial, porque a sua correção requer a eliminação das causas, para que assim se possa categorizar os fatores etiológicos em uma ordem que facilite o diagnóstico e o tratamento. Em todas as classificações sobre a etiologia das maloclusões, os hábitos orais foram mencionados como um agente em potencial para o desenvolvimento de maloclusões $(1,4,10,13,14,23,25,27)$.

A persistência de hábitos bucais deletérios em crianças acima de 3 anos aumenta significativamente a probabilidade do arco dental vir a apresentar características oclusais indesejáveis na dentição decídua $(4,25,27)$. Estes dados confirmam a importância da intensidade-freqüência-duração dos hábitos para o desenvolvimento e manutenção da maloclusão $(1,14)$.

Em conclusão, a prevalência de hábitos bucais foi elevada, existindo forte associação com o tipo de aleitamento e com a presença de maloclusão. Os resultados aqui verificados chamam atenção para a magnitude do problema na infância, enfatizando a necessidade da realização de estudos longitudinais, bem como a instituição de programas de atenção à saúde aos pré-escolares, envolvendo os pais ou responsáveis a fim de informá-los sobre os benefícios do aleitamento materno e alertá-los quanto aos efeitos deletérios que podem ser causados pelos hábitos de sucção não nutritiva • 
Agradecimentos. Os autores agradecem ao Conselho Nacional de Desenvolvimento Científico e Tecnológico (CNPq) pela concessão da Bolsa de Iniciação Científica. Expressam também o seu agradecimento à Priscila M. Bezerra pelo valoroso auxílio na coleta dos dados.

\section{REFERÊNCIAS}

1. Agurto PV, Diaz RM, Cadiz OD, Bobenrieth FK. Frecuencia de malos hábitos orales y su asociación con el desarrollo de anomalías dentomaxilares en niños de 3 a 6 años del área Oriente de Santiago. Rev Chil Pediatr 1999;70(6):470-482.

2. Serra-Negra JMC, Pordeus IA, Rocha Jr JF. Estudo da associação entre aleitamento, hábitos bucais e maloclusões. Rev Odontol Univ São Paulo 1997;11(2):79-86.

3. Valença AMG Vasconcelos FGG Cavalcanti AL, Duarte RC. Prevalência ecaracterísticas de hábitos orais em crianças. Pesqui Bras Odontoped Clin Integr 2001;1(1):1724.

4. Caglar E, Larsson E, Andersson EM, Hauge MS, Ogaard B, Bishara S, Warren J, Noda T, Dolci GS. Feeding, artificial sucking habits, and malocclusions in 3-year-old girls in different regions of the world. J Dent Child 2005;72(1):25-30.

5. Viggiano D, Fasano D, Monaco G Strohmenger L. Breast feeding, bottle feeding, and non-nutritive sucking; effects on occlusion in deciduous dentition. Arch Dis Child 2004;89(12):1121-1123.

6. Neiva FCB, Cattoni DM, Ramos JLA, Issler H. Desmame precoce: implicações para o desenvolvimento motor-oral. J Pediatr 2003;79(1):7-12.

7. Queluz DP, Gimenez CMM. Aleitamento e hábitos deletérios relacionados a oclusão. Rev Paul Odontol 2000;22(6):16-20.

8. Aarts C, Hornell A, Kylberg E, Hofvander Y, Gebre-Medhin M. Breastfeeding patterns in relation to thumb sucking and pacifier use. Pediatrics. 1999 Oct;104(4):e50.

9. Degan VV, Puppin-Rontani RM. Prevalence of pacifier-sucking habits and successful methods to eliminate them--a preliminary study. J Dent Child 2004;71(2):148151.

10. Calisti LJP, Cohen MM, Fales MH. Correlation between malocclusion, oral habits and socio-economic level of preschool children. J Dent Res 1960;39(5):450-454.

11. Ogaard B, Larsson E, Lindsten R. The effect of oral habits of sucking habits, cohort, sex, intercanine arch widths, and breast or bottle feeding on posterior crossbite in Norwegian and Swedish 3-year-old children. Am J Orthod Dentofacial Ortop 1994;106(2):161-166.

12. Shetty SR, Munshi AK. Oral habits in children-a prevalence study. J Indian Soc Pedod Prev Dent 1998;16(2):61-66.

13. Karjalainen S, Ronning O, Lapinleimu H, Simell O. Association between early wearning, on-nutritive sucking habits and occlusal anomalies in 3-year-old finnish children

Int J Paediatr Dent 1999;9(1):169-173. 
14. Chevitarese AB, Valle D, Moreira TC. Prevalence of malocclusion in 4-6 year old Brazilian children. J Clin Pediatr Dent 2002;27(1):81-85.

15. Charchut SW, Allred EN, Needleman HL. The effects of infant feeding patterns on the occlusion of the primary dentition. J Dent Child 2003;70(3):197-203.

16. Sousa FRN, Taveira GS, Almeida RVD, Padilha WWN. O aleitamento materno e sua relação com hábitos deletérios emaloclusão dentária. Pesqui Bras Odontopediatria Clín Integr 2004;4(3):211-216.

17. Ozawa N, Sakumittsu H, Takekoshi F, Shinji H. A study on non-nutritive sucking habits in young Japanese children-Relationships among incidence, duration, malocclusion and nursing behavior. Pediat Dent J 2005;15(1):64-71.

18. Lopez del Valle LM, Singh GD, Feliciano N, Machuca M. del C. Associations between a history of breast feeding, malocclusion and parafunctional habits in Puerto Rican children. P R Health Sci J. 2006 Mar;25(1):31-34.

19. Adair SM, Milano M, Lorenzo I, Russell C. Effects of current and former pacifier use on the dentition of 24- to 59-month-old children. Pediatr Dent 1995;17(7):437444.

20. Farsi NM, Salama FS. Sucking habits in Saudi children: prevalence, contributing factors and effects on the primary dentition. Pediatr Dent 1997;19(1):28-33.

21. Tomita NE, Bijella VT, Franco LJ. Relação entre hábitos bucais e maloclusão em préescolares. Rev Saúde Pública 2000;34(3):299-303.

22. Warren JJ, Bishara SE, Steinbock KL, Yonezu T, Nowak AJ. Effects of oral habits' duration on dental characteristics in in the primary dentition. J Am Dent Assoc 2001;132(12):1685-1693.

23. Katz CR, Rosenblatt A, Gondim PP. Nonnutritive sucking habits in Brazilian children: Effects on deciduous dentition and relationship with facial morphology. Am J Orthod Dentofacial Orthop 2004;126(1):53-57.

24. Instituto Brasileiro de Geografia e Estatística. [Sítio na Internet] IBGE Cidades. Disponível em: http://www.ibge.gov.br/cidadesat/default.php. Acesso em 25 de julho 2006.

25. Warren JJ, Bishara SE. Duration of nutritive and nonnutritive sucking behaviors and their effects on the dental arches in the primary dentition. Am J OrthodDentofacial Orthop 2002;121(4):347-356.

26. Chan SC, Tsai JS, King NM. Feeding and oral hygiene habits of preschool children in Hong Kong and their caregivers' dental knowledge and attitudes. Int J Paediatr Dent 2002;12(5):322-331.

27. Aznar T, Galán AF, Marin I, Domínguez A. Dental arch diameters and relationships to oral habits. Angle Orthodontist 2005;76(3):441-445.

28. Kruger E, Dyson K, Tennant M. Pre-school child oral health in rural Western Australia. Aust Dent J 2005;50(4):258-262. 